\title{
A Theoretical Review of Channel Coding Techniques Over Different Channel Models
}

\author{
Kulvir Kaur ${ }^{1}$, Heena Dogra ${ }^{2}$ and Gurjot Singh Gaba ${ }^{3 *}$ \\ 1,2,3 Discipline of Electronics and Communication Engineering \\ Lovely Professional University, Phagwara, Punjab, India - 144411 \\ ${ }^{1}$ sidhhukulvir18@gmail.com ,2heenadogra.007@gmail.com, \\ ${ }^{*}$ Corresponding Author - ${ }^{3}$ er.gurjotgaba@gmail.com
}

\begin{abstract}
The communication sector has noticed the effectiveness of wireless media for information exchange. The data exchange through wireless channel is affected by various impairments like interference, fading, noisy channel and attenuation. These effects have different impact on different wireless channel models. For an efficient and reliable communication, it is necessary to receive error free data. For error free communication, the channel coding is used. The aim of channel coding is to find an efficient code; which can be used for detection and correction of errors. In this paper, we have compared the channel coding techniques on the basis of Bit error rate over different channel models like Additive white Gaussian noise, Rician and Rayleigh channel model.
\end{abstract}

Keywords: Channel coding, BER, Channel model, $A W G N$

\section{Introduction}

Today, communication system demands higher data rates but this may lead to high BER (Bit Error Rate) if done by increasing the bandwidth. So, we do not consider the enlargement of bandwidth as an efficient solution [1]. The efficient coding techniques are there which helps us to achieve this goal. During transmission, the distortions occurred due to packet loss, errors etc. In this paper, we have considered the effective channel coding techniques to overcome these problems and also to increase the quality of transmission by decreasing the BER over different channel scenarios. The received signals in wireless communication have different time delays because of various effects like diffraction, refraction and scattering. The BER in these scenarios is high so the probability of packet loss is more. The BER is considered as main factor to evaluate the performance of wireless transmission. In wireless communication, the forward error correction techniques are used to reduce the BER. There is another factor which affects the BER, i.e. Signal to Noise ratio (SNR). These two terms are inversely proportional to each other if the SNR is more than the BER. SNR is determined by two terms:

$$
\begin{gathered}
\mathbf{E}_{\mathbf{b}}=\text { Error function } \\
\mathbf{N}_{\mathbf{o}}=\text { Noise power spectral density }
\end{gathered}
$$

In wireless communications, there are different channel models. The performance of a communication system in these channel models has a different behavior. Some basic channel models in wireless are AWGN (Additive White Gaussian Noise), Rayleigh, Rician and Nakagami. These channel models in terms of BER is improved by coding techniques like: Convolution coding, LDPC (low density parity check) coding, turbo codes etc. In this paper, we made a comparative analysis of these channel coding techniques over AWGN, Rayleigh and Rician channel models. The section I and II of the

Received (February 12, 2017), Review Result (August 9, 2017), Accepted (August 19, 2017)

* Corresponding Author 
manuscript explains the introductory concept of wireless communication, channel models and channel coding techniques. The comparative study of different channel coding techniques is analyzed in section III followed by section IV which contains the conclusion of this survey.

\section{Theoretical Analysis of Channel Modeling and Channel Coding Techniques}

There are various channel models and channel coding techniques which are taken into consideration. The theoretical analysis of those techniques has been carried out in this section:

\subsection{Channel Modeling}

In communication system, every signal requires a channel to reach towards destination. The channel is basically a bridge between transmitter and receiver. In wireless communication, there are various effects attenuation, fading, scattering, reflection etc. which degrades the performance of channel. To know the extent of losses, channel models are devised. Some basic channel models are discussed in the subsequent sub-section:

\subsubsection{AWGN Channel}

The additive white Gaussian noise is the basic model used in communication. In this model, the impairments arise only due to white noise, and therefore no traces of diffraction, scattering, attenuation etc. is observed [2]. That is why; this channel is considered as basic channel model.

The mathematical equation is:

$$
o(t)=i(t)+n(t)
$$

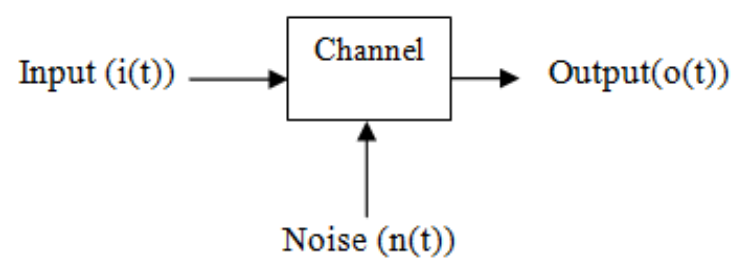

\section{Figure 1. Schematic of AWGN Channel Model}

Where $o(t)$ is received signal over AWGN channel, $i(t)$ is transmitted signal and $n(t)$ is background noise.

\subsubsection{Rayleigh Channel}

In Rayleigh fading channel, the received signal is affected by atmosphere and free space loss, this induces signal degradation and multipath propagation [3]. The environment which has multiple scatters simulated by Rayleigh model has no line of sight path. The result of this effect is that, the signal fluctuates in terms of phase and magnitude at receiver. This effect has more impact near the earth surface due to movable objects in between the sender and receiver, the frequency shifts. This effect is called as Doppler shift.

\subsubsection{Rician Channel}

The Rician fading channel is based on stochastic theory. In this model, there are many NLOS (non line of sight path) and at least one direct path [4]. When there is condition in 
which direct path has stronger effect then Rician fading occurs. It is based on two main parameters: $\mathrm{K}$ and $\Omega$ where $\mathrm{K}$ is strength of direct path and $\Omega$ is strength from all the paths [4].

\subsection{Channel Coding}

In order to avoid the unnecessary retransmissions which generate delay in process, the channel coding techniques are suggested [1]. The error detection and correction in transmission is usually done by various channel coding techniques. There are some significant channel coding techniques:

\subsubsection{Convolution Coding}

The convolution coding is an error correcting technique which add redundancy to original message bits and generates parity symbols by employing sliding window concept [6]. The receiver retrieves the only correct or most likely transmitted bits. As compared to linear block codes, the implementation of convolution codes is somewhat complex. But the tendency to correct the errors is more [6].

\subsubsection{Turbo Coding}

The turbo codes are using iterative soft decoders and recursive coders to eliminate the problems which occurred in block codes and convolution codes. In order to approach the Shannon limit the block codes have to use large block length and convolution codes have to use constraint length but recursive coders make the constraint length short and iterative soft decoders improves the estimation of received signal [5].

Path 1

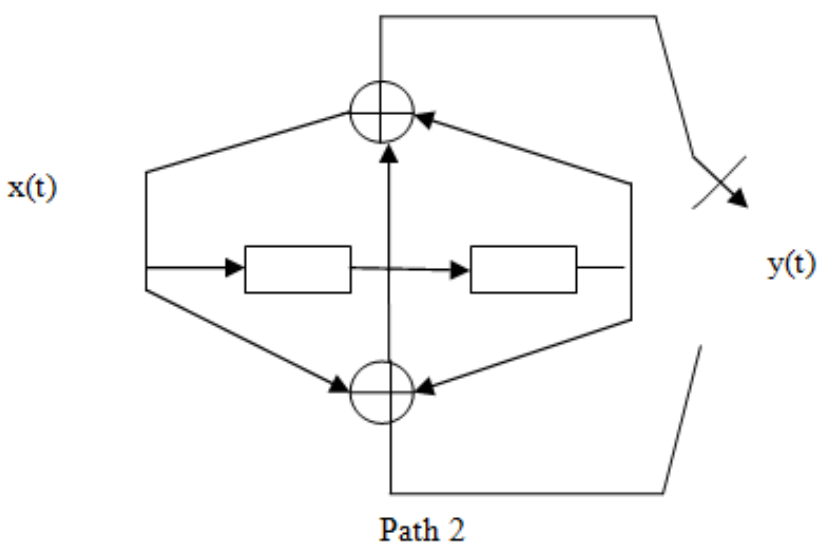

Figure 2. Convolution Encoding Circuit

\subsubsection{LDPC Coding}

These codes are linear error correcting codes. In this technique, the signal is transmitted on noisy channel but it is observed the signal suffers less loss of data. In recent years, these codes are able to attract more researches because of its various attributes like these codes are capacity approaching codes and the decoding process is also efficient when done with parallel iterative decoding methods. These codes proved themselves as efficient by performing close to Shannon limit. [7] 


\section{Performance of Channel Coding Techniques under Different Channel Models}

In this section, we have reviewed the performance of various channel coding techniques under different channel scenarios [8]. The performance of LDPC and turbo coding is analyzed over AWGN and Rayleigh fading channel and it has been concluded that LDPC code outperform the turbo code over flat Rayleigh fading channel. In recent studies, the LDPC codes are used instead of AWGN, Rayleigh, Rician and Nakagami channels and it has been observed that LDPC performs well and its code rate $1 / 2$ helps to achieve high gain with maintenance of spectral efficiency. In [9], the comparative analysis of different block codes over AWGN channel has been done and it has been shown that the LDPC has best performance over other coding techniques. The performance of turbo codes over AWGN, Rayleigh and Rician channel is analyzed and it proved that turbo codes are better than convolution coding for small frame size (for 500 bits) [10]. If LDPC codes are used for large frame size with sophisticated LDPC decoders, then it can provide $69.4 \%$ better result in terms of throughput without effecting error correction capabilities [11].

\section{Conclusion}

For reliable communication, the channel coding plays an important role as this technique makes the transmission error free by correcting the errors. The review presented in this paper contains the performance analysis of various coding techniques and it is observed that the LDPC (low density parity check) codes outperform all the other coding techniques. LDPC technique has simple implementation of encoder and decoder which makes way for researchers to study and improve. With AWGN channel, the LDPC code gives the excellent performance in comparison of other codes.

\section{References}

[1] J. S. Nandaniya, "Comparative Analysis of Different Channel Coding Techniques", International Journal of Computer Networks and Wireless Communications (IJCNWC), vol. 4, no. 2, (2014), pp. 84-89.

[2] M. S chavan and R. H. Chile, "Multipath Fading Channel Modeling and Performance Comparison of Wireless Channel Models", International Journal of Electronics and Communication Engineering, vol. 4, no. 2, (2011), pp. 189-203.

[3] S Pathak and H Katiyar, "Performance Analysis of Wireless link in Rician Fading Channel." International Journal of Advanced Research in Computer and Communication Engineering, vol. 5, no. 4, (2016), pp. 101-105.

[4] S. Golam, "Bit error rate (BER) comparison of AWGN channel for different type's digital modulation using MATLAB Simulink", American scientific research journal for engineering, technology and sciences (ASRJETS), vol. 13, no. 1, (2015), pp.61-71.

[5] E. K. Hall and S. G. Wilson, "Design and analysis of turbo codes on Rayleigh fading channels", in IEEE Journal on Selected Areas in Communications, vol. 16, no. 2, (1998), pp. 160-174..

[6] R. A. Baby, "Convolution coding and applications: A performance analysis under AWGN channel", 2015 International Conference on Communication Networks (ICCN), Gwalior, (2015), pp. 84-88.

[7] N. Kiyani and J. Weber, "Analysis of random regular LDPC codes on Rayleigh fading channels", 27th Symp. Inform. Theory Benelux.

[8] J. Hou, P. H. Siegel and L. B. Milstein, "Performance analysis and code optimization of low density parity-check codes on Rayleigh fading channels", in IEEE Journal on Selected Areas in Communications, vol. 19, no. 5, (2001), pp. 924-934.

[9] J. Singh and S. Bahel, "Comparative Study of Data Transmission Techniques of Different Block Codes over AWGN Channel using Simulink", International Journal of Engineering Trends and Technology (IJETT), vol. 9, no. 12, (2014), pp. 609-615.

[10] S. Papaharalabos, P. Sweeney and B. G. Evans, "A new method of improving SOVA turbo decoding for AWGN, Rayleigh and Rician fading channels", Vehicular Technology Conference, 2004. VTC 2004Spring. 2004 IEEE 59th, vol. 5, (2004), pp. 2862-2866.

[11] X. Zuo, I. Perez-Andrade, R. G. Maunder, B. M. Al-Hashimi and L. Hanzo, "Improving the Tolerance of Stochastic LDPC Decoders to Over clocking-Induced Timing Errors: A Tutorial and a Design Example”, in IEEE Access, vol. 4, (2016), pp. 1607-1629. 


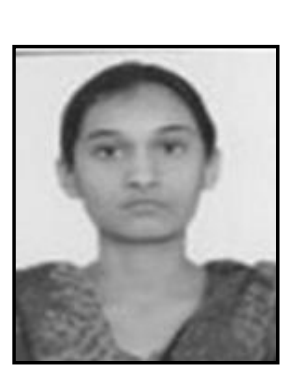

\section{Authors}

Kulvir Kaur, received the Bachelor's degree in Electronics and Communication from Lovely Professional University, Punjab. She is currently doing M. Tech in Communication Engineering. She has published one conference paper based on free space optical communication. Her field of interest includes free space optical communication in MIMO.

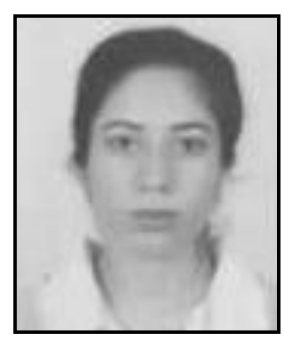

Heena Dogra, received the Bachelor's degree in Electronics and Communication from Himachal Pradesh Technical University. She is currently doing M.Tech in Communication engineering. Her field of interest is efficient Group Keying Security Techniques for Wireless sensor networks and Wireless Communications.

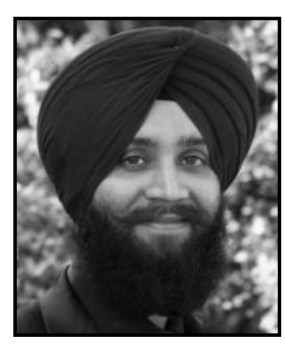

Gurjot Singh Gaba, is currently pursuing Ph.D. in Electronics \& Electrical Engineering with Spl. in Cryptography and Network Security of WSN and IoT's. He is working as an Asst. Prof. in Lovely Professional University, India since 2011. His research interest includes Wireless Sensor Networks, Computer Networks, Optical Communications and Cryptography. $\mathrm{He}$ is a reviewer of SCIE and Scopus Indexed Journals. He has recently been appointed as Editor of IJEEE journal. He is a member of many technical bodies including ISCA, IAENG, IACSIT, CSI, and ISTE. He is an author of six International books and more than two dozen research papers. 
International Journal of Control and Automation Vol.10, No.9 (2017) 